\title{
Apolipoprotein E2 Promotes the Migration and Invasion of Pancreatic Cancer Cells via Activation of the ERKI/2 Signaling Pathway
}

This article was published in the following Dove Press journal: Cancer Management and Research

\section{Hui Wang \\ Shaoxia Du \\ Jun Cai \\ Juan Wang \\ Xiaohong Shen}

School of Medicine, Nankai University, Tianjin 30007I, People's Republic of China
Correspondence: Xiaohong Shen School of Medicine, Nankai University, Nankai District, Tianjin 30007I, People's Republic of China

Tel +86-22-23501649

Email zebial2014@I63.com
Background: Apolipoprotein E2 (ApoE2) is reported to be essential for cell metastasis and proliferation and has been considered a potential diagnostic marker in many cancers. However, the function of ApoE2 in the metastasis of pancreatic cancer, as well as the underlying mechanism, remain unclear.

Purpose: In this study, we explored the effect of ApoE2 on the migration and invasion abilities of pancreatic cancer cells and explored the underlying molecular mechanism.

Methods and Results: Wound healing and Matrigel Transwell assays were used to investigate the role of ApoE2 in cell migration and invasion. Western blotting analysis showed that ApoE2 was overexpressed in pancreatic cancer tissues. Additionally, the overexpression of ApoE2 promoted the process of epithelial-mesenchymal transition (EMT) and enhanced the expression of MMP-2/9 in pancreatic cancer cells. Mechanistically, we found that inhibition of ERK1/2 signaling with PD98059 impaired the ApoE2-mediated promotion of cell migration, invasion and EMT.

Conclusion: This study demonstrated that ApoE2/ERK1/2 signaling promoted the migration and invasion of pancreatic cancer cells. ApoE2 might be a potential therapeutic target for the treatment of pancreatic cancer metastasis.

Keywords: apolipoprotein E2, ERK1/2, migration, invasion, pancreatic cancer

\section{Introduction}

Pancreatic ductal adenocarcinoma (PDAC) is a malignant tumor that occurs in the digestive tract and is associated with high mortality. ${ }^{1-3}$ The main reason for this high mortality is the strong metastatic ability of PDAC. ${ }^{4,5}$ Therefore, it is urgent to further reveal the underlying mechanisms of PDAC migration and invasion and to identify efficient biomarkers and therapeutic targets.

Recently, it was demonstrated that dyslipidemia is strongly implicated in the pathogenesis of malignant tumors. ${ }^{6-9}$ Apolipoprotein E (ApoE), a plasma protein, is a ligand for low-density lipoprotein receptors and participates in the circulation of cholesterol and other lipids. ${ }^{10}$ There are three main subtypes of ApoE: ApoE2, ApoE3 and ApoE4. ApoE2 and ApoE4, but not ApoE3, are closely related to abnormal blood lipid metabolism. ${ }^{11,12}$ Moreover, ApoE2 can enhance the vascular endothelial growth factor (VEGF)-induced migration of human retinal microvascular endothelial cells (HRMECs). ${ }^{13}$ Our previous study showed that the expression of ApoE2 in pancreatic cancer tissues is higher than that in adjacent normal tissues, induces cell proliferation and enhances G1/S and G2/M transition. ${ }^{14}$ 
Accumulating evidence has indicated that ApoE modulates the AKT, ERK1/2, and JNK signaling pathways in various processes, including cell metabolism, cell survival and hippocampal synaptic function. ${ }^{15-17}$ The MAPK/ ERK1/2 signaling pathway is an important intracellular pathway that regulates multiple cellular biological responses, such as cell growth, apoptosis, migration and invasion. ${ }^{18,19}$ The activation of this pathway promotes the expression of matrix metalloproteinases (MMPs), which in turn strengthen the degradation of the extracellular matrix and then promote cell movement. ${ }^{20}$ In addition, epithelialmesenchymal transition (EMT) has an important impact on tumor cell deformation and metastasis. ${ }^{21}$ Targeting MMPs and EMT is a promising strategy for inhibiting cancer cell metastasis. ${ }^{21}$ In our previous study, we demonstrated that ApoE2 promoted the phosphorylation of ERK1/2 by binding to the LRP-8 receptor in PDAC. ${ }^{14}$ Consequently, we hypothesized that ApoE2 might play a role in modulating PDAC cell migration and invasion by activating ERK1/2 signaling.

In this study, we explored the effects of ApoE2 on cell invasion, migration and EMT in human pancreatic cancer cells and determined the role of ERK1/2, AKT and JNK signaling in this process. We demonstrated that ApoE2 enhanced the migration and invasion abilities of pancreatic cancer cells by activating the ERK1/2 signaling pathway. Furthermore, we also showed that ApoE2 modulated the process of EMT and the expression of MMP-2/9 by activating the ERK1/2 signaling cascade.

\section{Patients and Methods}

\section{Patients and Sample Preparation}

Fresh pancreatic adenocarcinoma and adjacent tissue samples were collected from pancreatic cancer patients who underwent radical pancreatectomy without chemotherapy or radiation. This study involving human samples was approved by the Human Research Committee of Nankai University and the Chinese Anti-Cancer Association (CACA) and was conducted in accordance with the Declaration of Helsinki. Informed consent to participate in the study was obtained from the research subjects prior to study commencement.

\section{Cell Culture, Transfection and Treatment} The human pancreatic cancer cell lines AsPC-1, Capan-2 and PANC-1 were purchased from ATCC (Manassas, VA, USA). AsPC-1 and Capan-2 cells were grown in RPMI1640 medium, and PANC-1 cells were cultured in DMEM containing 10\% fetal bovine serum (FBS; BI, Australia). The normal human pancreatic ductal epithelial cell line (HPDE) H6c7 was maintained in keratinocyte serum-free medium (Gibco-BRL, Grand Island, NY). ${ }^{22}$ All the cells were cultured at $37^{\circ} \mathrm{C}$ with $5 \% \mathrm{CO} 2$ in a humidified incubator.

Small interfering RNAs (siRNAs) targeting ApoE2 were purchased from Synbio Tech (Shanghai, China). The pCMV6-ApoE2 plasmid (No. 01110-4b; Jiangsu, China) was obtained from the Protein and Plasmid Library. INTERFERin ${ }^{\circledR}$ transfection reagent (Polyplus, France) was used to transfect the cells with the pCMV6ApoE2 plasmid and siRNAs. Recombinant human ApoE2 (rhApoE2) was obtained from PeproTech (No. 350-12, Rocky Hill, New Jersey, USA). LY294002 (an inhibitor of PI3K), SP600125 (an inhibitor of JNK) and PD98059 (an inhibitor of ERK1/2) were acquired from Beyotime Biotechnology (China). The cells were pretreated with PD98059 $(30 \mu \mathrm{M})$, SP600125 $(20 \mu \mathrm{M})$ or LY294002 $(50 \mu \mathrm{M})$ for 1 hour.

\section{Cell Migration and Invasion}

Pancreatic cancer cells were seeded in 24-well plates. After cultivation overnight, the cells were transfected with the pCMV6-ApoE2 plasmid or siRNAs or treated with rhApoE2, PD98059, LY294002 or SP600125. After 24 hours, the treated cells were resuspended in $2 \%$ FBS and then seeded in the upper chambers of Transwell plates ( $8 \mu \mathrm{m}, \mathrm{BD}$ Bioscience, USA) with or without matrigel coating. Meanwhile, $600 \mu \mathrm{L}$ medium containing $10 \%$ fetal bovine serum was added to the lower chamber. The cells were cultured for 24 hours and 48 hours to assess migration and invasion, respectively. The cells in the upper chamber were removed with cotton swabs, and the cells in the lower chamber were fixed with anhydrous methanol and stained with crystal violet. The stained cells in five randomly selected fields were counted under 40x magnification.

\section{Wound-Healing Assays}

Cells were plated in 6-well plates. The next day, the cells were treated under different conditions. After 24 hours of treatment, a wound was generated by scraping with a $10 \mu \mathrm{L}$ pipette tip. For the next 48 hours, the cells were cultured with a cell proliferation inhibitor (LB-100) ${ }^{23}$ and pictures of the injured monolayer film were taken in the same fields every 6 hours. After measuring the width of at least four wounds, the relative closed wound distance was calculated. 


\section{Western Blotting Analysis}

RIPA lysis buffer (Sangon Biotech, Shanghai, China) was used to harvest the total protein from tissues and cell lines. After centrifugation and quantification, an equal volume of 1x loading buffer was added to the supernatant, and the supernatant was boiled for $10 \mathrm{~min}$ at $100^{\circ} \mathrm{C}$. Then, the protein samples were separated by SDS-PAGE and transferred to polyvinylidene difluoride membranes (Millipore, USA). Next, the membranes were blocked with $5 \%$ skim milk at room temperature for 1 hour and incubated at $4{ }^{\circ} \mathrm{C}$ overnight with the following primary antibodies: anti-MMP -2 (1:1000, Abcam, UK), anti-MMP-9 (1:1000, Abcam, UK), anti- $\beta$-actin $(1: 10,000)$, anti-E-cadherin $(1: 1000$, Abcam, USA), anti-N-cadherin (1:1000, CST, USA), and anti-Vimentin ( $1: 1000$, CST, USA). The next day, the membranes were incubated with rabbit/mouse secondary antibodies for 1 hour at room temperature. The immune complexes were visualized by an ECL reagent (Millipore, USA). The Western blotting bands were quantified by ImageJ software.

\section{Confocal Microscopy}

PANC-1 cells were seeded in 12-well plates. After overnight culture, the cells were transfected with the pCMV6ApoE2 plasmid or siRNAs. After 24 hours of treatment, the cells were digested and plated on coverslips that had been placed in 24-well plates. The cells were fixed for 15 min with anhydrous methanol and then blocked for 1 hour at room temperature with $10 \%$ goat serum. Then, the cells were incubated with anti-N-cadherin (N-cad), antiE-cadherin (E-cad) and anti-Vimentin (VIM) (diluted 1:200) antibodies at $4^{\circ} \mathrm{C}$ overnight, followed by incubation with a fluorescent secondary antibody for 1 hour at room temperature in the dark. Finally, the cells were stained with Hoechst 33,342 (1:2000) for $5 \mathrm{~min}$, washed with PBST three times, and placed on microscope slides. Images were acquired with a laser scanning microscope.

\section{Real-Time Quantitative PCR (RT-qPCR)}

The RNApure Tissue \& Cell Kit (CWBIO, China) was used for RNA extraction. Then, the HiFiScript 1st Strand cDNA Synthesis Kit (CWBIO, China) was used to reverse transcribe the total RNA into cDNA. GoTaq ${ }^{\circledR}$ qPCR Master Mix (Promega, USA) was used to perform RT-qPCR in a $25-\mu \mathrm{L}$ reaction volume, and the samples were detected by using iQSYBR-Green Supermix (Bio-Rad) in a CFX96 real-time PCR detection system (Bio-Rad). The data were analyzed by the $2^{-\Delta \Delta \mathrm{Ct}}$ method. The GAPDH levels served as the internal
Table I Primer Sets Used for RT-qPCR Analysis

\begin{tabular}{|l|l|}
\hline Gene & Primer Sequences (5' to 3', Forward to Reverse) \\
\hline E-cad & $\begin{array}{l}\text { Forward: 5'-CCTTCACAGCAGAACTAACA-3' } \\
\text { Reverse: 5'-CGCTTTCAGATTTTCATCAA-3' }\end{array}$ \\
\hline N-cad & $\begin{array}{l}\text { Forward: 5'-TGTTTGACTATGAAGGCAGTGG-3' } \\
\text { Reverse: 5'-TCAGTCATCACCTCCACCAT-3' }\end{array}$ \\
\hline VIM & $\begin{array}{l}\text { Forward: 5'-CAACGACAAAGCCCGCGTCG-3' } \\
\text { Reverse: 5'-GCGCAGGGCGTCATTGTTCC-3' }\end{array}$ \\
\hline MMP-2 & $\begin{array}{l}\text { Forward: 5'-TCAAGTTCCCCGGCGATG-3' } \\
\text { Reverse: 5'-AGTTGGCCACATCTGGGTTG-3' }\end{array}$ \\
\hline MMP-9 & $\begin{array}{l}\text { Forward: 5'-CGCTGGGCTTAGATCATTCC-3' } \\
\text { Reverse: 5'-TTGTCGGCGATAAGGAAGG-3' }\end{array}$ \\
\hline GAPDH & $\begin{array}{l}\text { Forward: 5'-TTGTCTCCTGCGACTTCA-3' } \\
\text { Reverse: 5'-CCACCACCCTGTTACTGTT-3' }\end{array}$ \\
\hline
\end{tabular}

reference. The primers used in this study were purchased from Sangon Biotech (Shanghai, China). Table 1 lists the primer sequences of the target genes.

\section{Gelatin Zymography}

Cells were seeded in 6-well plates and transfected with the ApoE2 plasmid and siApoE2. Forty-eight hours after transfection, MMP activity in the supernatants was determined using gelatin zymography. In brief, $80 \mu \mathrm{g}$ protein of each sample was separated by SDS-PAGE gel containing $0.1 \%$ $(\mathrm{w} / \mathrm{v})$ gelatin without a reducing agent. Then, the gels were washed for 40 min with $2.5 \%$ (w/v) Triton X-100 and then mixed slowly at $37^{\circ} \mathrm{C}$ for 24 hours in the incubation solution (200 mM NaCl, $5 \mathrm{mM} \mathrm{CaCl} 2,0.02 \%$ (w/v) Brij-35 and 50 $\mathrm{mM}$ Tris- $\mathrm{HCl}(\mathrm{pH} 7.6)$ ). Finally, $0.5 \%$ Coomassie Brilliant Blue R-250 (Sigma) was used to stain the gels for 2-3 hours. The gels were then washed three times with decolorizing liquid for $30 \mathrm{~min}$. The clear areas on the gels represent the activity of the MMPs.

\section{Animal Experiments}

A nude mouse experiment was performed to assess in vivo tumour metastasis ability of PANC-1 cells stably expressing ApoE2 or empty vector. These cells $\left(5 \times 10^{6}\right.$ cells $)$ were inoculated into the abdominal cavity of 5-week-old BALB/ c athymic nude mice (Beijing HFK Bio-Technology, Beijing, China), five mice per group. All animal studies were approved by the Ethics Committee of the Nankai University and conducted by skilled experimenters under an approved protocol in accordance with the principles and procedures outlined in the NIH Guide for the Care and Use 
of Laboratory Animals. Numbers of visible metastases in the gut and liver, as well as invisible micrometastases, were counted after mice were sacrificed. ${ }^{24}$

\section{Statistical Analysis}

The data obtained from three independent experiments are expressed as the mean \pm standard deviation (SD). The experimental groups and the control group were compared using Student's $t$-test and ANOVA. $P<0.05$ was considered statistically significant.

\section{Results}

\section{ApoE2 Was Overexpressed in Pancreatic Cancer Tissues and PANC-I Cells}

To determine whether ApoE2 affects the development of pancreatic cancer, we first detected the expression level of ApoE2 in pancreatic cancer tissue samples. As shown in Figure $1 \mathrm{~A}$ and $\mathrm{B}$, the expression levels of ApoE2 in pancreatic cancer tissues were higher than those in paracarcinoma tissues, as detected by RT-qPCR and Western blotting. Additionally, we assessed the expression levels of ApoE2 in pancreatic cancer cell lines (AsPC-1, Capan-2 and PANC-1) and HPDE cells (H6C7) by Western blotting and RT-qPCR. The results showed that ApoE2 was mainly expressed in PANC-1 cells (Figure 1C). These results indicate that ApoE2 plays an important role in the occurrence and development of pancreatic cancer.

\section{ApoE2 Promoted the Migration and Invasion Abilities of Pancreatic Cancer Cells}

To investigate the role of ApoE2 in the migration and invasion of pancreatic cancer cells, ApoE2 was overexpressed in AsPC-1, Capan-2 and PANC-1 cells by rhApoE2 treatment or pCMV6-ApoE2 transfection. ApoE2 expression was significantly increased in cells following transfection with pCMV6-ApoE2 but decreased in cells following transfection with siApoE2 (Figure 2A). Then, we performed Matrigel Transwell analysis, and the results showed that ApoE2 overexpression markedly

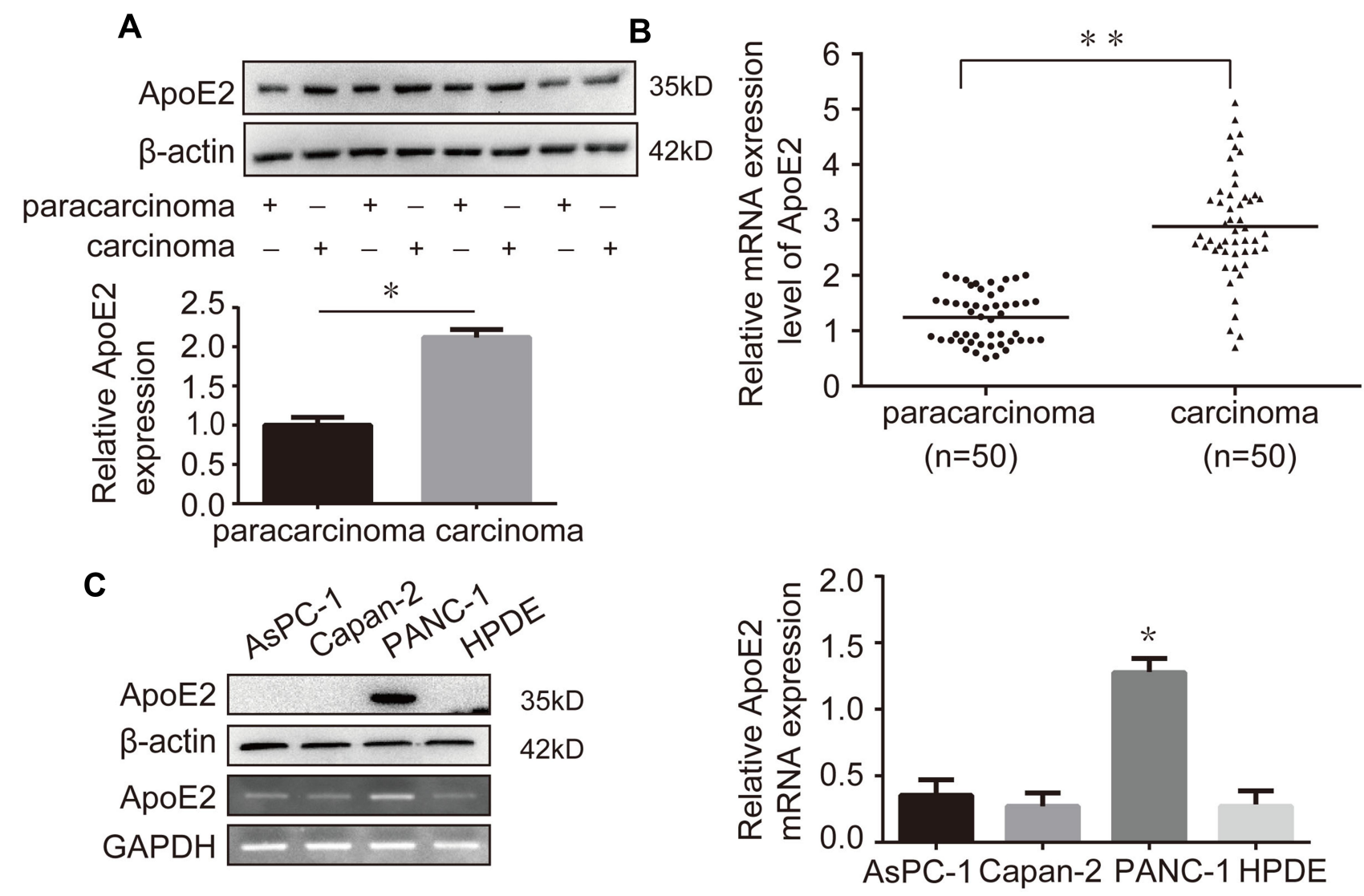

Figure I ApoE2 was overexpressed in pancreatic cancer tissues. (A) Western blotting analysis was performed to detect the expression levels of ApoE2 in 4 paired pancreatic cancer tissues and paracarcinoma tissues. (B) The mRNA level of ApoE2 in 50 paired pancreatic cancer tissues and paracarcinoma tissues was examined by RTqPCR. (C) The expression levels of ApoE2 in pancreatic cancer cell lines (AsPC-I, Capan-2 and PANC-I) were measured by Western blotting and RTqPCR $(* P<0.05, * * P<0.01)$. 


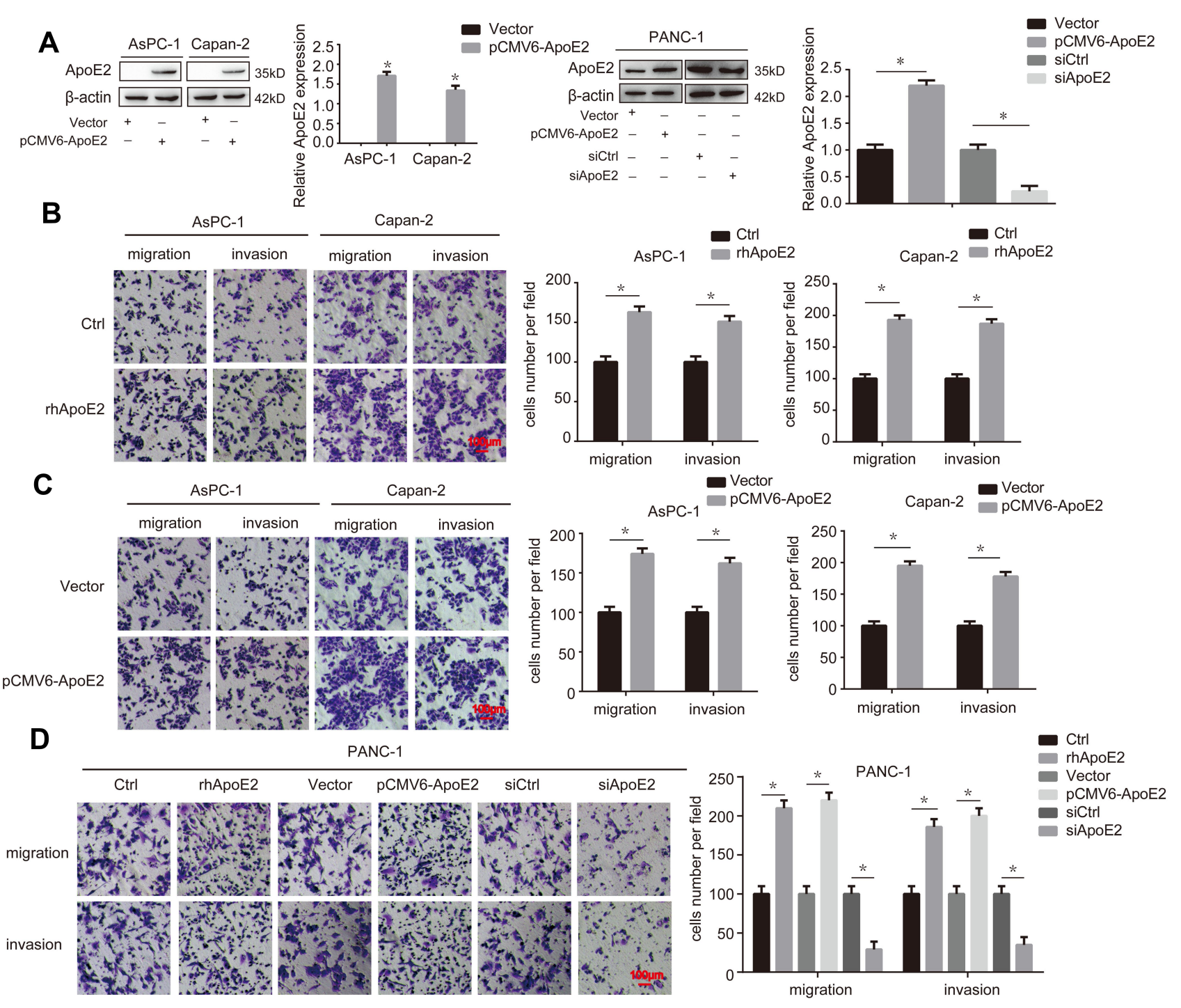

Figure 2 ApoE2 promoted the migration and invasion abilities of pancreatic cancer cells. (A) The transfection efficiencies of siApoE2 and PCMV6-ApoE2 in AsPC-I, Capan2 and/or PANC-I cells were detected by Western blotting. (B) A Matrigel Transwell assay was used to determine the function of rhApoE2 in AsPC-I and Capan-2 cell migration and invasion. (C) A Matrigel Transwell assay was used to assess the role of pCMV6-ApoE2 in AsPC-I and Capan-2 cell migration and invasion. (D) The effects of rhApoE2, pCMV6-ApoE2 and siApoE2 on migration and invasion in PANC-I cells were determined by Matrigel Transwell assay. ( $n=3$, *P<0.05).

increased the migration and invasion abilities of AsPC-1, Capan-2 and PANC-1 cells (Figure 2B-D). The Matrigel Transwell analysis results showed that ApoE2 depletion significantly reduced the migration and invasion abilities of PANC-1 cells (Figure 2D). All the data showed that ApoE2 contributed to the migration and invasion potential of pancreatic cancer cells.

\section{ApoE2 Modulated the Expression of EMT Markers and MMP-2/9}

To determine the potential mechanism by which ApoE2 induced the migration and invasion of pancreatic cancer cells, we examined the expression of tumor metastasis- related genes in AsPC-1, Capan-2 and PANC-1 cells by Western blotting and immunofluorescence analysis. Figure $3 \mathrm{~A}$ and $\mathrm{B}$ show that the overexpression of ApoE2 by rhApoE2 treatment or pCMV6-ApoE2 transfection increased the expression of $\mathrm{N}$-cadherin (N-cad) and Vimentin (VIM) but decreased the expression of E-cadherin (E-cad) in AsPC-1 and Capan-1 cells. Moreover, the expression level of MMP-2/9 was increased following rhApoE2 treatment or pCMV6-ApoE2 transfection (Figure $3 \mathrm{~A}$ and $\mathrm{B}$ ). In PANC-1 cells, rhApoE2 and pCMV6-ApoE2 also increased the expression of $\mathrm{N}$-cad, VIM and MMP-2/9 and decreased the expression of E-cad, and knockdown of ApoE2 caused the opposite result (Figure $3 \mathrm{C}$ ). To further confirm the results described above, we also 


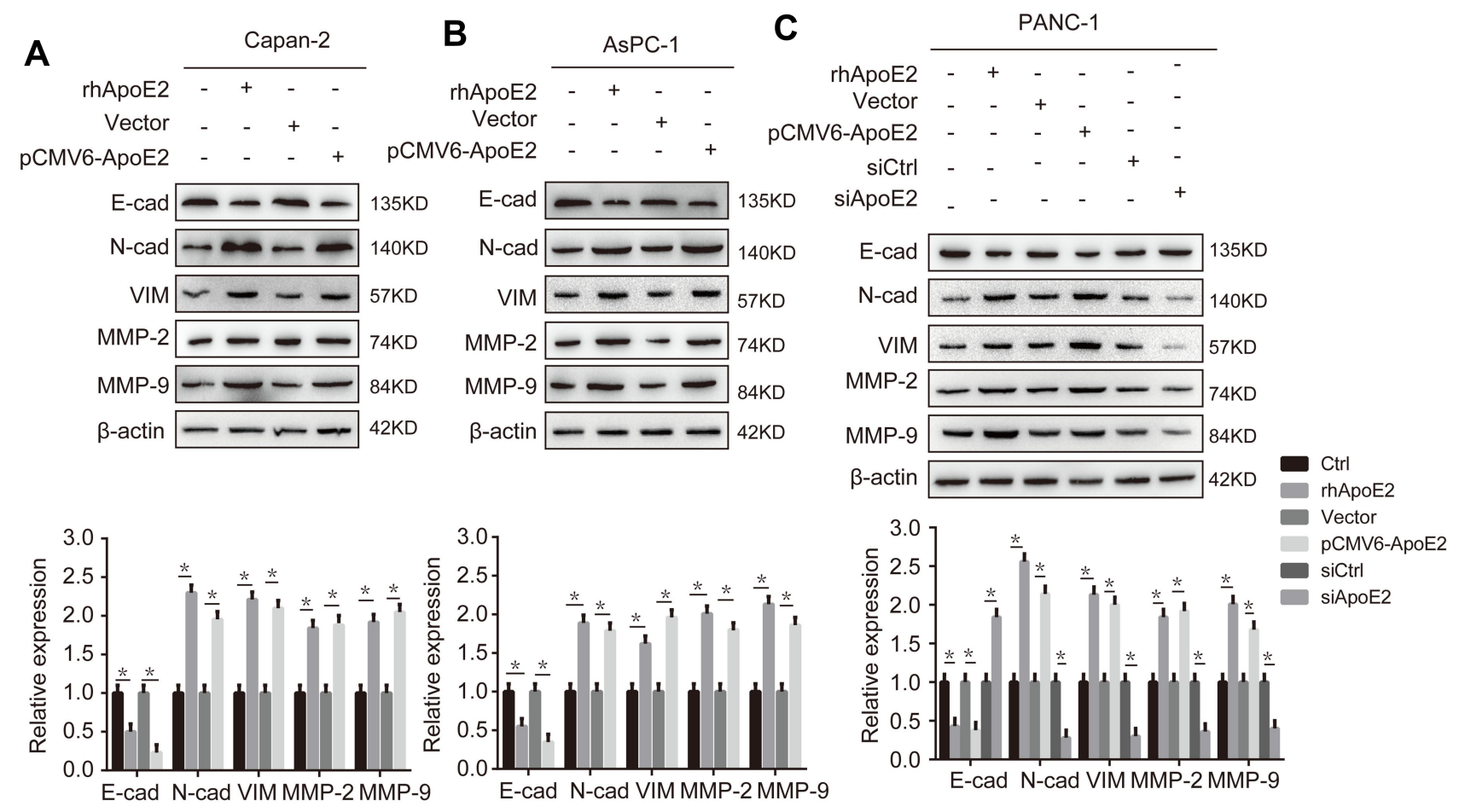

Figure 3 ApoE2 facilitated EMT and induced MMP-2/9 expression in pancreatic cancer cells. (A) Western blotting analysis was performed to detect the expression of N-cad, E-cad, VIM and MMP-2/9 in Capan-2 cells treated with rhApoE2 or transfected with pCMV6-ApoE2. (B) The expression of E-cad, N-cad, VIM and MMP-2/9 in AsPC-I cells treated with rhApoE2 or transfected with PCMV6-ApoE2 was examined by Western blotting. (C) The expression of N-cad, E-cad, VIM and MMP-2/9 in PANC-I cells treated with rhApoE2 or transfected with PCMV6-ApoE2 or siApoE2 was detected by Western blotting. $(n=3, * P<0.05)$.

Abbreviations: N-cad, N-cadherin; E-cad, E-cadherin; VIM, Vimentin.

detected the expression levels of EMT markers (N-cad, E-cad, and VIM) by using immunofluorescence. Consistent with the Western blotting results, the overexpression of ApoE2 increased the expression of N-cad and VIM and decreased the expression of E-cad in PANC-1 cells (Figure 4A and B). All of these results indicated that ApoE2 facilitated the process of EMT and increased the expression of MMP-2/9 in pancreatic cancer cells.

\section{ApoE2 Promoted Cell Migration and Invasion Through Activation of ERKI/2 Signaling in PANC-I Cells}

Several reports have indicated that ApoE modulate the AKT, ERK1/2, and JNK signaling pathways in various cellular processes. ${ }^{15-17}$ Our previous study demonstrated that ApoE2 could activate ERK1/2 signaling in pancreatic cancer cells. ${ }^{14}$ In the present study, we explored whether the AKT, ERK1/2 and JNK signaling pathways were involved in the ApoE2-mediated promotion of the cell migration and invasion of PANC-1 cells. We found that of the three kinases mentioned above, the level of phosphorylated ERK1/2 was significantly increased in a time- dependent manner after treatment of the cells with rhApoE2 (Figure 5A). Then, inhibitors of AKT, ERK1/2 and JNK were used to inhibit the activation of the signaling pathways described above. As shown in Figure 5B, the levels of p-ERK1/2, p-AKT and p-JNK were inhibited by PD98059, LY294002 and SP600125, respectively. In addition, PD98059 significantly weakened the effect of ApoE2 in promoting the migration and invasion of PANC-1 cells, while LY294002 and SP600125 showed no significant effect, as detected by using the Transwell and woundhealing assays (Figure $5 \mathrm{C}$ and $\mathrm{D}$ ). These results demonstrated that ApoE2 contributed to cell migration and invasion via activation of ERK1/2 signaling in PANC-1 cells.

\section{ApoE2 Modulated the Expression of EMT Markers and MMP-2/9 by Regulating ERKI/2 Signaling}

We further explored the molecular mechanism by which ApoE2 modulates the EMT process. The Western blotting and RT-qPCR results showed that ApoE2 upregulated the $\mathrm{N}-\mathrm{cad}$ (CDH2) and VIM expression levels while 


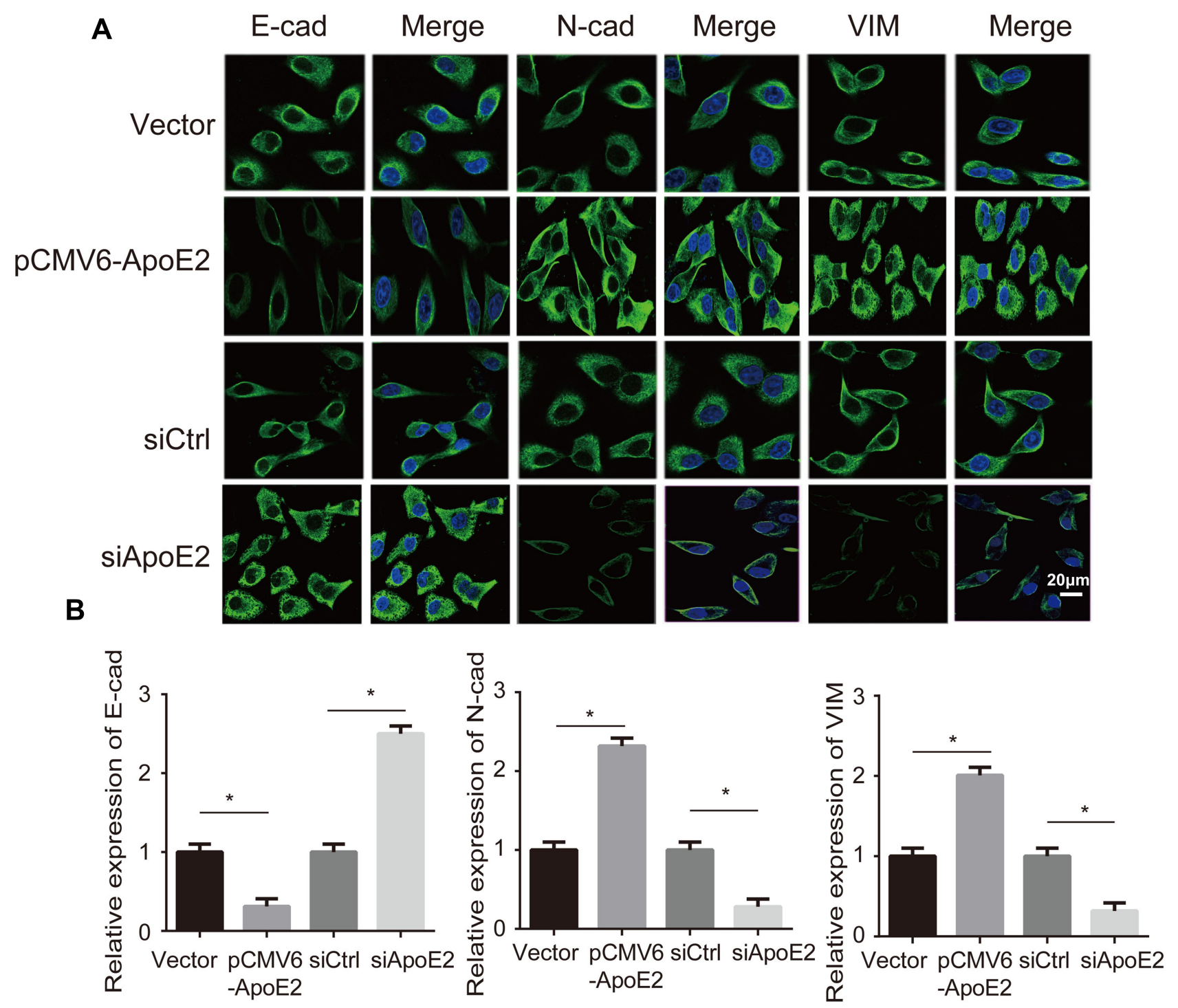

Figure 4 Immunofluorescence analysis of the protein expression of EMT markers in PANC-I cells with different treatments. (A) The expression of E-cad, N-cad and VIM in PANC-I cells transfected with PCMV6-ApoE2 or siApoE2, as well as their controls, was examined by confocal microscopy. The green signal represents the expression of $\mathrm{N}$-cad, E-cad and VIM, and the blue signal represents nuclear DNA stained with Hoechst 33,342. (B) The fluorescence intensity was determined and normalized to the cell background. The levels of N-cad, E-cad and VIM were calculated as a ratio compared to the controls. $(n=3, * P<0.05)$.

Abbreviations: N-cad, N-cadherin; E-cad, E-cadherin; VIM, Vimentin.

decreasing the E-cad (CDH1) expression level in PANC-1 cells, whereas PD98059 significantly reversed these trends (Figure 6A and B). As shown in Figure 6C, the overexpression of ApoE2 significantly increased the mRNA expression of MMP-2/9 in PANC-1 cells, while this ApoE2-induced effect was inhibited by PD98059. Gelatin zymography showed that ApoE2 stimulated the enzyme activity of MMP-2/9, whereas this ApoE2-induced increased activity was inhibited by PD98059 (Figure 6D). Consequently, these results demonstrated that ApoE2 regulated the expression of EMT markers and MMP-2/9 by regulating ERK1/2 signaling.
Furthermore, the nude mouse intraperitoneal tumor metastasis experiments showed that injection of ApoE2 overexpression-PANC-1 cells induced the numbers of abdominal metastasis nodules (Figure 6E). These data indicate that ApoE2 is a critical factor to induce development and metastasis in pancreatic cancer.

\section{Discussion}

As one of the most malignant forms of cancer, PDAC is characterized by high metastasis. ${ }^{4,5}$ Further exploration of the potential mechanism of tumor metastasis is helpful for improving therapeutic efficacy. In the current study, we 


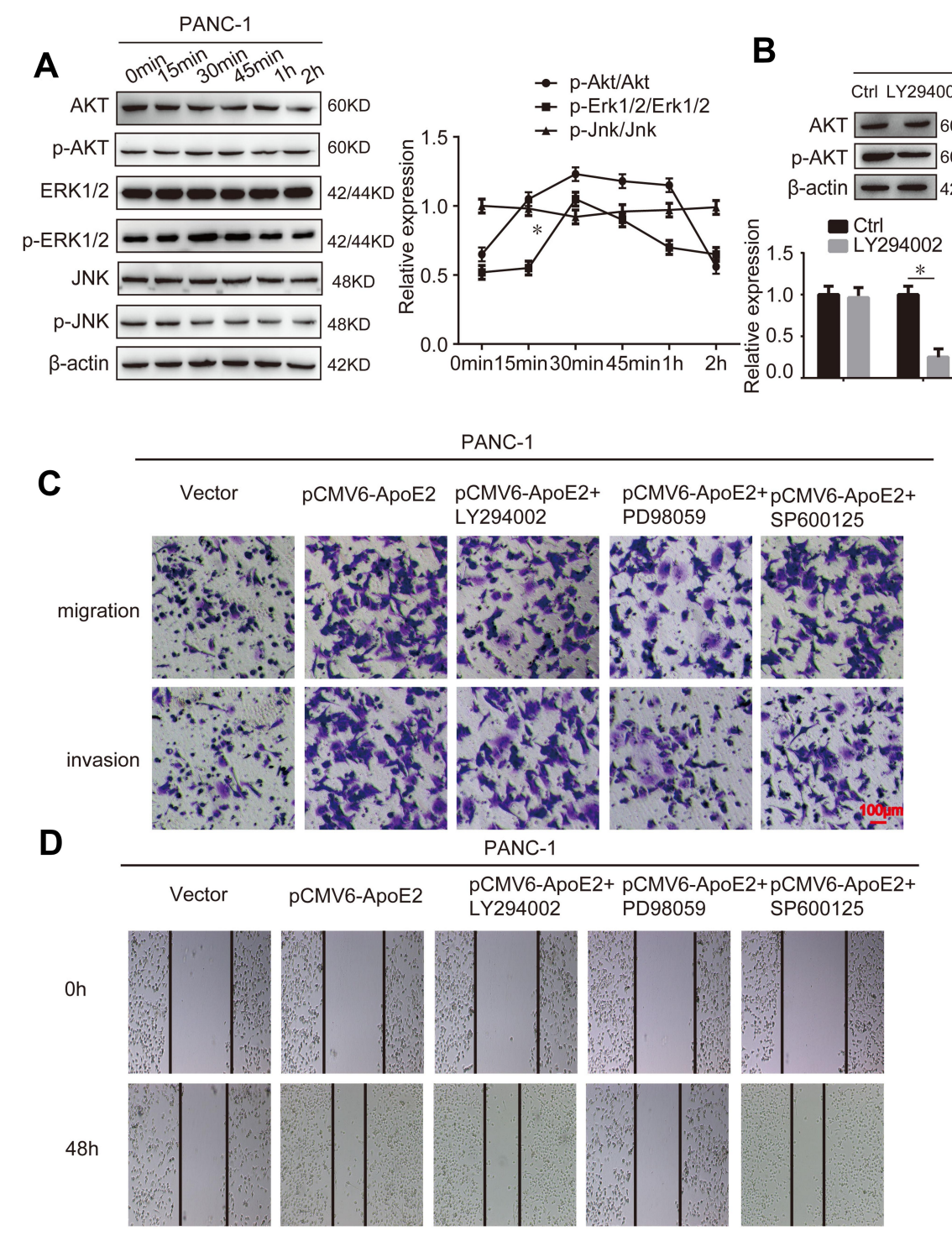

PANC-1

Ctrl LY294002 Ctrl PD98059 $\quad$ Ctrl SP600125

$\mathrm{AKT}=-60 \mathrm{KD} \quad \mathrm{ERK} 1 / 2 \longrightarrow 42 / 44 \mathrm{KD} \quad \mathrm{JNK}-\longrightarrow 48 \mathrm{~K}$

p-AKT $\longrightarrow$ 60KD p-ERK1/2 - $242 / 44 K D$ p-JNK $-48 K D$

$\beta$-actin $=42 \mathrm{KD} \quad \beta$-actin $\longrightarrow$ 42KD $\beta$-actin $\longrightarrow$ 42KD
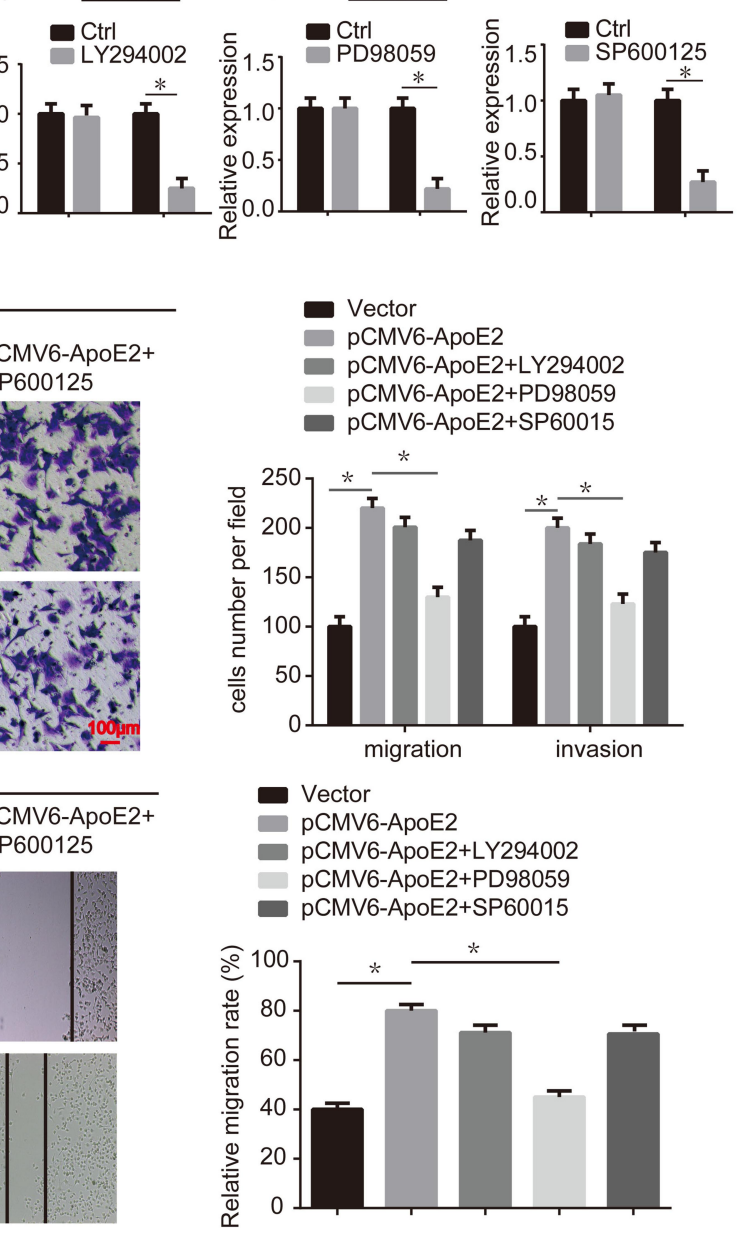

Figure 5 ApoE2 promoted cell migration and invasion via activation of ERKI/2 signaling in PANC-I cells. (A) The expression of AKT, p-AKT, ERKI/2, p-ERKI/2, JNK and P-JNK was detected by Western blotting after cells were treated with rhApoE2 $(200 \mathrm{ng} / \mathrm{mL})$ for different durations. (B) The efficiencies of the inhibitors were detected by Western blotting. (C) The migration and invasion abilities of PANC-I cells were determined by using a Matrigel Transwell assays after treatment or transfection of the cells. (D) A wound-healing assay was used to determine the migration ability of PANC-I cells with different treatments. The data represent the mean \pm SD $(n=3$, $* P<0.05$ ).

demonstrated that ApoE2 facilitated the metastasis and invasion of pancreatic cancer by activating ERK1/2 signaling.

Previously, it has been reported that ApoE participates in various biological cellular processes, such as cell migration, proliferation and immune regulation. ${ }^{25-28}$ In vitro assays have shown that ApoE2 induces cell proliferation and migration in HRMECs. ${ }^{13}$ Additionally, we found that ApoE2 was overexpressed in pancreatic cancer tissues, and further results indicated that ApoE2 was essential for cell proliferation and cell cycle progression in pancreatic cancer cells. ${ }^{14}$ In this study, we explored the role of ApoE2 in pancreatic cancer migration and invasion and the underlying mechanism. We found that ApoE2 overexpression induced significant enhancements in the cell migration and invasion capacities of pancreatic cancer cells and accelerated the EMT process. Our results illustrated that ApoE2 might be a potentially effective target for the treatment of PDAC, especially for the treatment of PDAC metastasis.

In addition, we explored the molecular mechanism by which ApoE2 regulates the metastasis of pancreatic cancer cells. In our previous study, we found that ApoE2 could enhance the phosphorylation of ERK $1 / 2$ by binding to the LRP-8 receptor. ${ }^{14}$ Growing evidence has demonstrated that the PI3K/AKT, ERK1/2 and JNK signaling pathways 


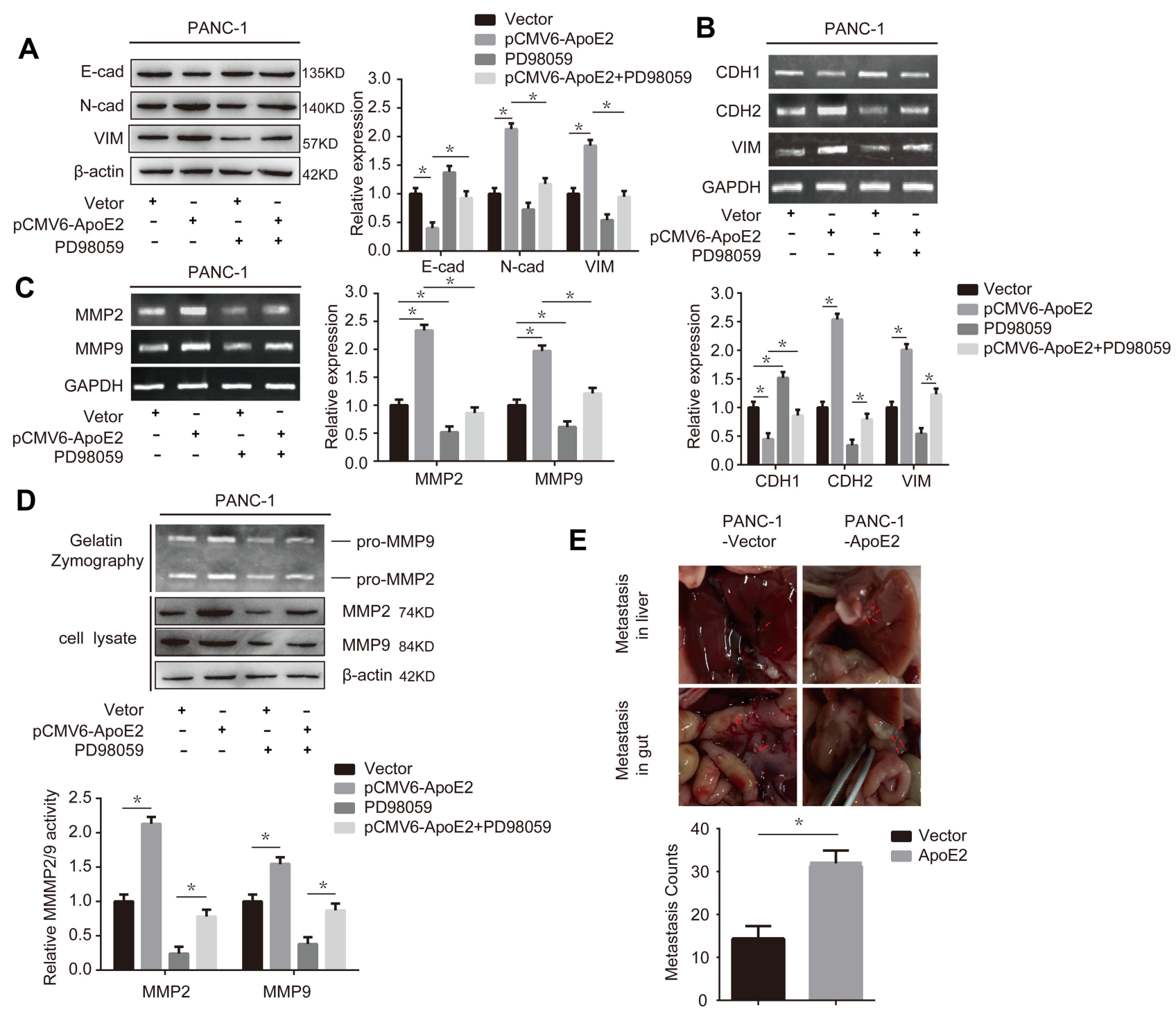

Figure 6 ApoE2 modulated the expression of EMT markers and MMP-2/9 by regulating ERKI/2 signaling. (A) PANC-I cells were treated with PD98059 before transfection with PCMV6-ApoE2 or the control vector. (A) After 48 hours, the protein levels of E-cad, N-cad and VIM were assessed by Western blotting. (B) The mRNA levels of E-cad, N-cad and VIM in the indicated cells were measured by RT-qPCR. (C) The MMP activity was analyzed by RT-qPCR. (D) The MMP activity was analyzed by gelatin zymography (D-top) and Western blotting (D-bottom). $(n=3, * P<0.05)(C D H I, E$-cadherin; CDH2, N-cadherin; VIM, Vimentin). (E) Nude mouse intraperitoneal tumor cell metastasis assay. The total numbers of visible metastatic lesions in abdominal cavity are presented.

are strongly implicated in the migration and invasion of various kinds of cancers, including PDAC. ${ }^{29-34}$ In this study, we determined the effects of LY294002, PD98059 and SP600125 on the ApoE2-induced migration and invasion of PANC-1 cells. We found that PD98059, but not LY294002 or SP600125, significantly impaired the effect of ApoE2 in promoting the cell migration and invasion of pancreatic cancer.

Tumor metastasis is a complex biological process that involves multiple factors, stages and genes. Recent studies have shown that the EMT program is closely linked to tumor metastasis. ${ }^{35,36}$ Notably, EMT markers, including
E-cad (marker of epithelial cells), N-cad and VIM (markers of mesenchymal cells), are regulated by the ERK1/2 signaling pathway in pancreatic cancer. ${ }^{37-40}$ In the current study, we indicated that ApoE2 activated ERK1/2 signaling to promote the expression of N-cad and VIM at both the mRNA and protein levels while reducing the expression of E-cad.

Moreover, we demonstrated that ApoE2 enhanced the mRNA and protein expression levels of MMP-2/9 by activating the ERK1/2 signaling pathway. Invasion refers to the behavior by which cancer cells break through the basement membrane to infiltrate the surrounding tissues; 
degradation of the basement membrane and extracellular matrix (ECM) contributes to cancer cell invasion. MMP-2/ 9 are important ECM kinases and play vital roles in ECM degradation. ${ }^{41-43}$ It has been shown that ERK1/2 signaling activation can induce MMP-2/9 expression. ${ }^{44}$ Consistently, we observed that treatment with PD98059 decreased the expression of MMP-2/9 induced by ApoE2 in PANC-1 cells.

In summary, this study reveals that ApoE2 effectively induces the progression of EMT in pancreatic cancer cells and stimulates the secretion of activated MMP-2/9 through the ERK1/2 signaling pathway to enhance the invasion and migration capacities of pancreatic cancer cells. Our study suggests that ApoE2 might be a potential therapeutic target for treating the metastasis and invasion of pancreatic cancer.

\section{Acknowledgments}

The present study was funded by the National Natural Fund (31370861).

\section{Disclosure}

All the authors state that they have no conflicts of interest.

\section{References}

1. Hidalgo M. Pancreatic cancer. $N$ Engl J Med. 2010;362 (17):1605-1617. doi:10.1056/NEJMra0901557

2. Siegel R, Naishadham D, Jemal A. Cancer statistics, 2013. $C A$ Cancer J Clin. 2013;63(1):11-30. doi:10.3322/caac.21166

3. Jemal A, Bray F, Center MM, et al. Global cancer statistics. $C A$ Cancer J Clin. 2011;61(2):69-90. doi:10.3322/caac.20107

4. Stathis A, Moore MJ. Advanced pancreatic carcinoma: current treatment and future challenges. Nat Rev Clin Oncol. 2010;7(3):163-172. doi: $10.1038 /$ nrclinonc. 2009.236

5. Schneider G, Siveke JT, Eckel F, et al. Pancreatic cancer: basic and clinical aspects. Gastroenterology. 2005;128(6):1606-1625. doi:10. 1053/j.gastro.2005.04.001

6. Snijder MB, Zimmet PZ, Visser M, et al. Independent and opposite associations of waist and hip circumferences with diabetes, hypertension and dyslipidemia: the AusDiab study. Int $J$ Obes Relat Metab Disord. 2004;28(3):402-409. doi:10.1038/sj.ijo.0802567

7. Yao X, Tian Z. Dyslipidemia and colorectal cancer risk: a meta-analysis of prospective studies. Cancer Causes Control. 2015;26(2):257-268. doi:10.1007/s10552-014-0507-y

8. Khaidakov M, Mitra S, Kang B-Y, et al. Oxidized LDL receptor 1 (OLR1) as a possible link between obesity, dyslipidemia and cancer. PLoS One. 2011;6(5):e20277. doi:10.1371/journal.pone.0020277

9. Ostroumova MN, Kovalenko IG, Bershteǐn LM, Tsyrlina EV, Dil'man VM. Characteristics of dyslipidemia in cancer patients. Vopr Onkol. 1986;32(1):34-43.

10. Yencilek F, Yilmaz SG, Yildirim A, et al. Apolipoprotein E genotypes in patients with prostate cancer. Anticancer Res. 2016;36(2):707-711.

11. Dallongeville J, Lussier-Cacan S, Davignon J. Modulation of plasma triglyceride levels by apoE phenotype: a meta-analysis. J Lipid Res. 1992;33(4):447-454.
12. Phillips MC. Apolipoprotein $\mathrm{E}$ isoforms and lipoprotein metabolism. IUBMB Life. 2014;66(9):616-623. doi:10.1002/iub.1314

13. Masuda T, Shimazawa M, Hashimoto Y, et al. Apolipoprotein E2 and $\mathrm{E} 3$, but Not E4, promote retinal pathologic neovascularization. Invest Ophthalmol Vis Sci. 2017;58(2):1208-1217. doi:10.1167/iovs.1620539

14. Du S, Wang H, Cai J, et al. Apolipoprotein E2 modulates cell cycle function to promote proliferation in pancreatic cancer cells via regulation of the c-Myc-p21 Waf1 signalling pathway. Biochem Cell Biol. 2020;98(2):191-202. doi:10.1139/bcb-2018-0230

15. Laffont I, Takahashi M, Shibukawa Y, et al. Apolipoprotein E activates Akt pathway in neuro-2a in an isoform-specific manner. Biochem Biophys Res Commun. 2002;292(1):83-87. doi:10.1006/ bbrc.2002.6586

16. Gan HT, Tham M, Hariharan S, et al. Identification of ApoE as an autocrine/paracrine factor that stimulates neural stem cell survival via MAPK/ERK signaling pathway. J Neurochem. 2011;117(3):565-578. doi:10.1111/j.1471-4159.2011.07227.x

17. Korwek KM, Trotter JH, LaDu M, et al. ApoE isoform-dependent changes in hippocampal synaptic function. Mol Neurodegener. 2009;4(1):21. doi:10.1186/1750-1326-4-21

18. Yu H, Zhu B, Yang W, et al. Matrine inhibits proliferation and migration of HepG2 cells by downregulating ERK1/2 signaling pathways. J Cancer Res Ther. 2020;16(2):209-214. doi:10.4103/ jcrt.JCRT_331_19

19. Qiang Y, Ma F, Wang Z, et al. LukS-PV induces cell cycle arrest and apoptosis through p38/ERK MAPK signaling pathway in NSCLC cells. Biochem Biophys Res Commun. 2020;521(4):846-852. doi:10. 1016/j.bbrc.2019.10.181

20. Chen YJ, Wei -Y-Y, Chen H-T, et al. Osteopontin increases migration and MMP-9 up-regulation via alphavbeta3 integrin, FAK, ERK, and NF-kappaB-dependent pathway in human chondrosarcoma cells. J Cell Physiol. 2009;221(1):98-108. doi:10.1002/jcp.21835

21. Gutschalk CM, Yanamandra AK, Linde N, et al. GM-CSF enhances tumor invasion by elevated MMP $-2,-9$, and -26 expression. Cancer Med. 2013;2(2):117-129. doi:10.1002/cam4.20

22. Liu N, Furukawa T, Kobari M, et al. Comparative phenotypic studies of duct epithelial cell lines derived from normal human pancreas and pancreatic carcinoma. Am J Pathol. 1998;153(1):263-269. doi:10.10 16/S0002-9440(10)65567-8

23. Bai X, Zhi X, Zhang Q, et al. Inhibition of protein phosphatase $2 \mathrm{~A}$ sensitizes pancreatic cancer to chemotherapy by increasing drug perfusion via HIF-1alpha-VEGF mediated angiogenesis. Cancer Lett. 2014;355(2):281-287. doi:10.1016/j.canlet.2014.09.048

24. Chen S, Chen J-Z, Zhang J-Q, et al. Hypoxia induces TWIST-activated epithelial-mesenchymal transition and proliferation of pancreatic cancer cells in vitro and in nude mice. Cancer Lett. 2016;383(1):73-84. doi:10.1016/j.canlet.2016.09.027

25. Ito Y, Takano T, Miyauchi A. Apolipoprotein e expression in anaplastic thyroid carcinoma. Oncology. 2006;71(5-6):388-393. doi:10. $1159 / 000107112$

26. Chen YC, Pohl G, Wang T-L, et al. Apolipoprotein E is required for cell proliferation and survival in ovarian cancer. Cancer Res. 2005;65 (1):331-337.

27. Huang DY, Weisgraber KH, Strittmatter WJ, et al. Interaction of apolipoprotein $\mathrm{E}$ with laminin increases neuronal adhesion and alters neurite morphology. Exp Neurol. 1995;136(2):251-257. doi:10.1006/ exnr.1995.1101

28. Kattan OM, Kasravi FB, Elford EL, et al. Apolipoprotein E-mediated immune regulation in sepsis. J Immunol. 2008;181(2):1399-1408. doi:10.4049/jimmunol.181.2.1399

29. You Q, Xu J, Zhu Z, et al. Phloretin flavonoid exhibits selective antiproliferative activity in doxorubicin-resistant gastric cancer cells by inducing autophagy, inhibiting cell migration and invasion, cell cycle arrest and targeting ERK1/2 MAP pathway. J BUON. 2020;25 (1):308-313. 
30. Li X, Ma N, Zhang Y, et al. Circular RNA circNRIP1 promotes migration and invasion in cervical cancer by sponging miR-629-3p and regulating the PTP4A1/ERK1/2 pathway. Cell Death Dis. 2020;11(5):399. doi:10.1038/s41419-020-2607-9

31. Li J, Yang C, Yang J, et al. Down-regulation of CCL17 in cancer-associated fibroblasts inhibits cell migration and invasion of breast cancer through ERK1/2 pathway. Cancer Manag Res. 2019;11 (p):7439-7453. doi:10.2147/CMAR.S211651

32. Xin B, He X, Wang J, et al. Nerve growth factor regulates CD133 function to promote tumor cell migration and invasion via activating ERK1/2 signaling in pancreatic cancer. Pancreatology. 2016;16 (6):1005-1014. doi:10.1016/j.pan.2016.09.005

33. Ni S, Wang H, Zhu X, et al. CBX7 suppresses cell proliferation, migration, and invasion through the inhibition of PTEN/Akt signaling in pancreatic cancer. Oncotarget. 2017;8(5):8010-8021. doi:10.18 632/oncotarget.14037

34. Wei W, Li H, Li N, et al. WNT5A/JNK signaling regulates pancreatic cancer cells migration by phosphorylating paxillin. Pancreatology. 2013;13(4):384-392. doi:10.1016/j.pan.2013.05. 008

35. Zhao H, Diao C, Wang X. LncRNA BDNF-AS inhibits proliferation, migration, invasion and EMT in oesophageal cancer cells by targeting miR-214. J Cell Mol Med. 2018;22(8):3729-3739. doi:10.1111/ jcmm. 13558

36. Su C, Cheng X, Li Y, et al. MiR-21 improves invasion and migration of drug-resistant lung adenocarcinoma cancer cell and transformation of EMT through targeting HBP1. Cancer Med. 2018;7(6):2485-2503. doi:10.1002/cam4.1294
37. Li S-L, Chen X, Wu T, et al. Knockdown of TMPRSS3 inhibits gastric cancer cell proliferation, invasion and EMT via regulation of the ERK $1 / 2$ and PI3K/Akt pathways. Biomed Pharmacother. 2018;107(p):841-848. doi:10.1016/j.biopha.2018.08.023

38. Wu L, Han L, Zhou C, et al. TGF-beta1-induced CK17 enhances cancer stem cell-like properties rather than EMT in promoting cervical cancer metastasis via the ERK1/2-MZF1 signaling pathway. FEBS J. 2017;284(18):3000-3017. doi:10.1111/febs.14162

39. Zhang M, Song S, Yi Z, et al. Human biliverdin reductase promotes EMT through the ERK1/2 signal pathway in breast cancer. Eur $J$ Pharmacol. 2016;788:45-53. doi:10.1016/j.ejphar.2016.06.019

40. Yang Y, Yang C, Zhang J. C23 protein meditates bone morphogenetic protein-2-mediated EMT via up-regulation of Erk1/2 and Akt in gastric cancer. Med Oncol. 2015;32(3):76. doi:10.1007/s12032-015-0547-5

41. Cao D, Fan ST, Chung SSM. Identification and characterization of a novel human aldose reductase-like gene. J Biol Chem. 1998;273 (19):11429-11435. doi:10.1074/jbc.273.19.11429

42. Luo Y, Liang F, Zhang ZY. PRL1 promotes cell migration and invasion by increasing MMP2 and MMP9 expression through Src and ERK1/2 pathways. Biochemistry. 2009;48(8):1838-1846. doi:10.1021/bi8020789

43. Rucci N, Sanita P, Angelucci A. Roles of metalloproteases in metastatic niche. Curr Mol Med. 2011;11(8):609-622. doi:10.2174/ 156652411797536705

44. Peng SY, Hsiao CC, Lan TH, et al. Pomegranate extract inhibits migration and invasion of oral cancer cells by downregulating matrix metalloproteinase-2/9 and epithelial-mesenchymal transition. Environ Toxicol. 2020;35(6):673-682. doi:10.1002/tox.22903

\section{Publish your work in this journal}

Cancer Management and Research is an international, peer-reviewed open access journal focusing on cancer research and the optimal use of preventative and integrated treatment interventions to achieve improved outcomes, enhanced survival and quality of life for the cancer patient.
The manuscript management system is completely online and includes a very quick and fair peer-review system, which is all easy to use. Visit http://www.dovepress.com/testimonials.php to read real quotes from published authors. 